\title{
Scheduling Scientific Workflows to Meet Soft Deadlines in the Absence of Failure Models ${ }^{\star}$
}

\author{
Kassian Plankensteiner, Radu Prodan, and Thomas Fahringer \\ Institute of Computer Science, University of Innsbruck, \\ Technikerstr. 21a, 6020 Innsbruck, Austria \\ \{kassian.plankensteiner, radu,tf $\} @ d p s$.uibk.ac.at
}

\begin{abstract}
Highly distributed systems such as Clouds and Grids are used to execute complex scientific workflow applications by researchers from various areas of science. While scientists rightfully expect efficient and reliable execution of their applications, current systems often cannot deliver the required Quality of Service. We propose a dynamic execution and scheduling heuristic able to schedule workflow applications with a high degree of fault tolerance, while taking into account soft deadlines. Experimental results show that our method meets soft deadlines in volatile highly distributed systems in the absence of historic failure trace data or complex failure models of the target system.
\end{abstract}

\section{Introduction}

The workflow paradigm has emerged as one of the most successful paradigms for execution of scientific applications on highly distributed systems such as Clouds, computational Grids or meta-computers. While scientists rightfully expect efficient and reliable execution of their applications on these systems, current systems often cannot deliver the Quality of Service (QoS) needed for widespread acceptance as ubiquitous scientific tools [10. The problem lies in the complex and highly dynamic nature of highly distributed systems, which exhibit high failure rates that the systems have to be able to cope with [6].

In real-life, users typically want to know an estimation of the execution time for their application before deciding to have it executed. In many cases, this estimation can be considered to be a soft deadline, defined as the requirement that the execution time satisfies a certain deadline with some probability $\alpha$ [16]. In other words, if a soft deadline is not met, the results are still useful, the only difference being in the QoS provided by the system. If a system regularly fails to meet soft deadlines by large amounts, users will be dissatisfied with the Quality of Service offered by the system. If a hard deadline is broken, there is no need for the results at all. Weather forecasting and medical simulations are two areas with typical hard deadlines.

\footnotetext{
* This work is partially funded by the European Union, grant agreement number 261585 (SHIWA project).
} 
In previous work, we proposed the Resubmission Impact (RI) heuristic [9] for scheduling workflows in faulty environments in the absence of failure models, and showed that it can provide a high degree of fault tolerance with a significantly reduced resource consumption compared to other common techniques. In this paper, we build on top of this heuristic a new method and algorithm to meet soft deadlines for workflows in highly distributed systems without failure models.

The paper is organised as follows. Section 2 discusses related work. Section 3 summaries previous work on the Resubmission Impact heuristic, which forms the foundation for our work in this paper. In Section 4 we introduce a new dynamic scheduling heuristic for meeting soft deadlines without a failure model. We present experimental results in Section 5 and conclude in Section 6 .

\section{Related Work}

Considering faults in the scheduling and execution of a workflow application, we can divide existing techniques into three broad categories.

The first category of techniques [715] build on (often extensive and complex) failure models that aim to describe the failure probability of a task on a resource in a certain time interval. Building such a model can be a very difficult task that often requires years worth of traces of failure data about the specific target environment. On the other hand, commercial Cloud providers such as Amazon do not disclose any information regarding their infrastructure at all, and failure traces are often a closely guarded secret.

The second category of techniques 251314 tackle the problem with the help of Service Level Agreements (SLAs) or Advance Reservations (AR). Techniques in this category usually rely on agreements with the end user as well as the resource providers, describing the QoS requirements for the execution of the given workflow. The workflow execution is accepted and started only if binding agreements with the resource providers can be reached. Given the strict criteria of QoS in the SLAs, this shifts the responsibility of dealing with faults to the resource providers. The problem with this approach is the lack of support for negotiating the required fine-grained QoS terms in todays Cloud/Grid systems.

The third category of techniques can often be found in current Grid workflow systems. As described in our earlier work on fault tolerance in [10, they often deal with the unreliability of the environments by resorting to fault tolerance mechanisms such as task replication and task resubmission without considering a failure model of the target system. This method has the advantage of applicability to new and unknown distributed environments, where no suitable failure model is available. Unfortunately, it often leads to unnecessarily large resource consumption and to large differences of the expected execution time (as promised to the end user) and the real execution time of a workflow and therefore degrades the Quality of Service. Our work in this paper belongs to this third category. It offers better QoS than many of the existing approaches in this category by foreseeing possible deadline violations at runtime and taking appropriate measures to positively influence the execution of the remainder of the workflow. 


\section{Resubmission Impact}

Resubmission Impact (RI) 9] is a heuristic for scheduling workflow applications in the presence of faults. RI is based on the idea of finding a compromise solution that balances the tradeoffs between resource consumption when using replication and the increase in execution time when using resubmission, by establishing a metric that describes the impact of the resubmission of a task on the workflow makespan.

Let $T$ denote the set of tasks in a workflow and $E$ the set of control flow and data flow dependencies between tasks. Let $\phi_{i} \in \Phi$ denote the amount of work (i.e. instructions) that each task $t_{i} \in T$ requires to be completely processed. Additionally, we define $\Psi$ to be a set of integer values defining the replication sizes $\psi_{i} \in \Psi$ for each task $t_{i} \in T$. Initially, we have $\forall \psi_{i} \in \Psi: \psi_{i}=0$. A workflow $\Gamma$ is defined as a 4-tuple: $\Gamma=(T, E, \Phi, \Psi)$. We also define $\Theta$ to be the total set of distributed resources available, and $r e p l_{\max }$, res $s_{\max }$ as the maximum replication count and maximum resubmission count, respectively. Both repl $l_{\max }$ and $r e s_{\max }$ have to be specified by the user as input. The RI heuristic can be seen in Algorithm 2 and consists of two phases.

The first phase (lines 1-7) establishes the resubmission impact metric. First, we make a copy $\Phi^{\prime}$ of the set $\Phi$ defining the work amount of each task in the workflow. Then, we enlarge the amount of work $\phi_{i}^{\prime}$ in task $t_{i}$ by multiplying it with the resubmission count $r e s_{\max }$, and define a new workflow $\Gamma^{\prime}$ containing the new work amount for each task. Afterwards, we compute the difference in expected execution time $\delta_{i}$ of $\Gamma$ and $\Gamma^{\prime}$, by scheduling both of them using the HEFT 11 algorithm (line 5). We repeat these steps for every task $t_{i} \in T$. Finally, we compute the resubmission impact $\lambda_{i}$ for every task $t_{i}$ by normalising $\delta_{i}$ against the maximum of all $\delta_{j}, j=0 \ldots n$ (line 7 ). The resubmission impact $\lambda_{i}$ reflects the impact of having to the resubmit task $t_{i}$ the given amount of times on the expected execution time of the whole workflow.

In the second phase of the RI algorithm (lines $8-11$ ), we create a copy of the replication count set $\Psi$ and call it $\Psi^{\prime}$. For every $\psi_{j}^{\prime} \in \Psi^{\prime}$, we set $\psi_{j}^{\prime}$ to be $\left\lfloor\lambda_{j} \cdot r e p l_{\text {max }}\right\rfloor$ (line 9). This defines the replication count for every task $t_{j} \in T$. Finally, we replace the set of replication counts $\Psi \in \Gamma$ with the newly defined $\Psi^{\prime}$ and send it to HEFT_replication 9 for scheduling.

HEFT_replication (Algorithm 1) is an extension to the Heterogeneous Earliest Finish Time (HEFT) [11] algorithm. It works in two phases: in the first phase (line $1-3$ ), it computes an upward rank for all the tasks based on the length of the critical path to the end of the workflow (also called the B-level), and sorts them based on the rank. In the second phase (line $4-14$ ), it maps the tasks in descending order of their ranks to the resource giving the earliest expected completion time (ECT). After each task is mapped, its parent tasks are checked (line $7-12$ ). If all child tasks have been mapped to a resource already, the previously set number of replicas of the parent are created and mapped to resources according to the earliest expected completion time. 

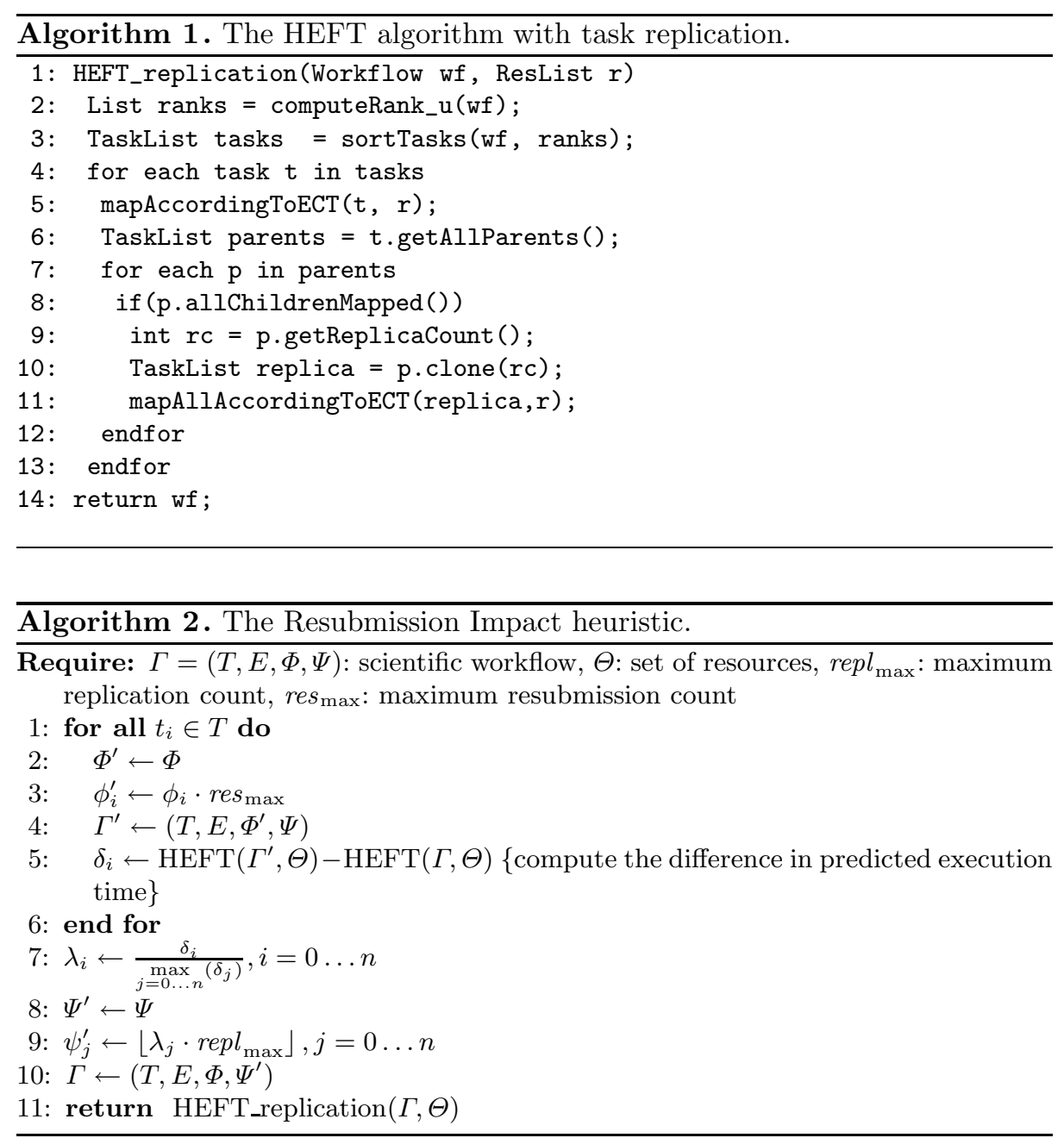

\section{Scheduling for Soft Deadlines}

The RI heuristic is able to schedule workflows with a high degree of fault tolerance while consuming a reasonably low amount of resources. However, since RI schedules the full workflow before it is executed, it cannot react to unexpected environment behaviour like periods of high failure occurrences. This can lead to situations where the replication approach can fail, and many tasks have to fall back to the resubmission technique. Consequently, the application runtimes can exhibit large deviations from the value expected immediately after scheduling, degrading the QoS for the end user.

To this end, we propose an extension to the RI heuristic that is able to provide an estimation of the execution time in the form of a soft deadline to the end 
user. To achieve this, we apply changes to RI in the following areas: (1) execution control by introducing a monitoring step after the completion of each task and rescheduling the workflow remainder if the soft deadline is likely to be missed; (2) scheduling by introducing a rescheduling heuristic that is able to influence the parameters of the fault tolerance strategy of RI, therefore decreasing the likelihood of a violation of the soft deadline.

\subsection{Execution Control}

Execution Control systems relying on pure full-ahead schedulers such as the RI heuristic are unable to readjust their fault tolerance strategy at runtime if the high fault rate makes the initially chosen replication amount ineffective. Once this happens, the execution systems have to fall back to resubmission, which leads to spikes in the workflow makespans that exceed the expected execution times often by large amounts, deteriorating the QoS for the end-user.

To solve this issue, we propose a dynamic execution control strategy as illustrated in Algorithm 3. We start by sending the workflow to the scheduler (line 1), which uses the RI heuristic to map tasks to computational resources and assigns to every task an expected start time and an expected finish time. Based on the schedule, we also obtain the expected execution time of the workflow and generate a soft-deadline for the execution (line 2 ). The workflow soft-deadline $t_{d}$ is computed as follows:

$$
t_{d}=t_{s} \cdot\left(1+r \cdot \sum_{n=1}^{\zeta_{\max }} \frac{1}{n}\right)
$$

where $t_{s}$ is the expected execution time as determined by the initial schedule of the workflow, $r$ is the rescheduling threshold, and $\zeta_{\max }$ is the maximum (re)scheduling count. The rescheduling threshold $r>0$ is a percentage given by the user that defines how much the real execution time is allowed to exceed the initial schedule before the first rescheduling cycle is started. The maximum (re)scheduling count $\zeta_{\max }>1$ defines the upper limit to the number of scheduling cycles to be performed for a given workflow during runtime, and is also given by the user. In line 3 , we compute the initial value for the rescheduling threshold time $t_{r}$ as follows:

$$
t_{r}=t_{s} \cdot \sum_{n=1}^{\zeta} \frac{r_{i}}{n},
$$

where $\zeta$ is the current scheduling cycle count (i.e. $\zeta=1$ for the initial cycle).

We enter the execution control loop in line 4 and start with the workflow execution by identifying the tasks that are ready to execute according to the current state of the workflow. Every ready task is sent to its corresponding resource based on the mapping done by the scheduler. Once the ready tasks are submitted, we wait for finished tasks to arrive back at the execution control component (line 8). As finished tasks return to the executor (line 9), we include a monitoring step that decides if we need a rescheduling operation. We compare the real finish time of the tasks to their planned finish time as calculated 


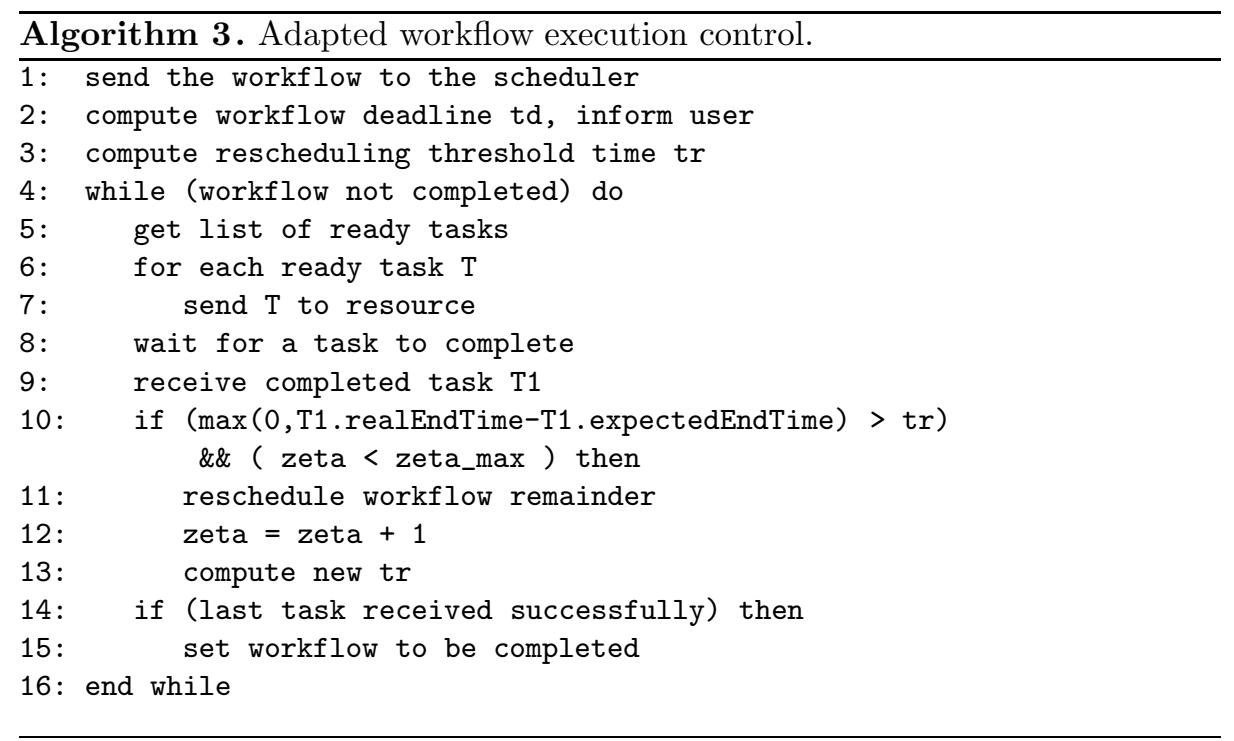

by the initial scheduling step. If the time difference is larger than the current rescheduling threshold time $t_{r}$, we invoke a rescheduling cycle for the remainder of the workflow (see Section 4.2), compute a new rescheduling threshold time by using Equation 2, and increment the scheduling cycle count $\zeta$ (line 11-13). Since every rescheduling cycle increases the amount of replication in the rest of the workflow, it is no longer invoked if the scheduling cycle count reaches $\zeta_{\max }$ to avoid excessive resource usage.

\subsection{Rescheduling}

As explained in the previous section, the new rescheduling heuristic is invoked if the execution control component discovers that the real execution of the workflow application is too far behind the scheduled workflow execution plan, which implies a high probability of missing the soft-deadline as given to the user. The job of the rescheduling heuristic is to decrease the probability of violating a deadline by adjusting the amount of replication, $\psi_{j}^{\prime}$, of each remaining task, $t_{j}$, of a workflow as follows:

$$
\psi_{j}^{\prime}=\left\lfloor\lambda_{j}^{\frac{1}{\zeta}} \cdot r e p l_{\max }\right\rfloor
$$

where $\lambda_{j}$ and $r e p l_{\max }$ are the resubmission impact of a task $t_{j} \in T$ and the maximum replication count, respectively.

Figure 1 shows the effect of using the term $\lambda_{j} \frac{1}{\zeta}$ in Equation 3 . We can see that the higher the scheduling cycle $\zeta$ is, the higher the replication count becomes for tasks with a smaller resubmission impact. In other words, with every rescheduling cycle we start replicating tasks that have not yet been replicated and increase the replication count on tasks that are already subject to replication. We base 


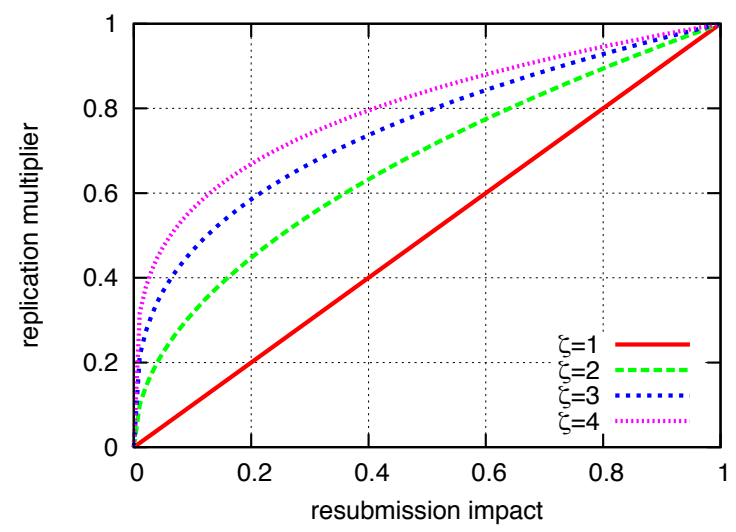

Fig. 1. Replication multiplier

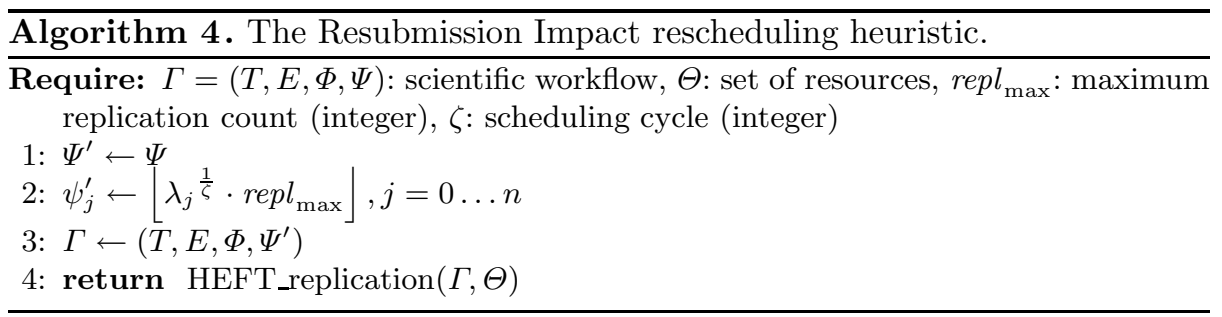

this on the resubmission impact $\lambda$ of a task because it is a measure of the impact of rescheduling that task on the global execution time of a workflow.

Algorithm 4 shows the proposed rescheduling heuristic, which in line 2 uses Equation 3 to adjust the replication count $\psi$ of the tasks in the workflow. Finally, HEFT_replication (see Algorithm 1) is used to schedule the remaining workflow tasks including the replicas.

\section{$5 \quad$ Experiments}

To evaluate our method we implemented a workflow management system based on the GridSim 3] Grid simulator, capable of scheduling and executing workflows according to Algorithms 3 and 4 .

\subsection{Resource Failure Model}

In a recent study, Iosup et al. 6] show that the mean time between failure (MTBF) for clusters in existing large-scale Grid environments can be modeled using a Weibull distribution. We utilised their findings to create a resource failure model for our simulated environment which considers the MTBF, the size of a failure in terms of the number of processors affected, as well as the duration of a 
failure (or mean time to recover (MTTR)). Thus, according to [6], we model the MTBF with a Weibull distribution having a shape parameter ranging between 11.5 and 12.5, the duration of a failure using a LogNormal distribution and the size of a failure using a Weibull distribution with a shape parameter between 1.5 and 2.4 .

\subsection{Simulation Setup}

Our simulated heterogeneous resource environment comprises 5480 processors with different computational power distributed over twelve clusters. We generated failure models for each of the twelve clusters based on the Weibull distributions for MTBF and failure size, and based on the LogNormal distribution for the MTTR, as described in Section 5.1. To study the impact of resource reliability on our (re)scheduling algorithm, we varied the Weibull scale parameter to create three different failure models based on different MTBF values: five hours for shaky resources, three days for the normal (average case) resources, and one week for stable resources.

We generated three types of workflows modeled after three real-world scientific workflow applications: Wien2K [1, a scientific workflow for performing electronic structure calculations of solids; Meteo $A G$, a workflow application designed for meteorological simulations based on the RAMS [4 numerical atmospheric model; POV-Ray 8, a movie-rendering workflow based on the popular open source Persistence of Vision raytracer. For each application type, we created 50 workflows based on randomly generated input problem sizes. Our experimental suite therefore consists of 150 different workflows with a wide range of computational tasks, task lengths, data transfer sizes and estimated execution times (from a few hours to longer than one month). The workflows are created based on historical performance data logged from real workflow runs executed in the Austrian Grid [12] during the past few years. We set $r=0.2$, res $s_{\max }=3$ and two different values for $r e p_{\max }, 2$ and 4 , to study the impact of the maximum replication count on our new technique. To compare the behaviour of our new scheduling method to our previous method of full-ahead scheduling with RI, we investigate a maximum scheduling cycle count $\zeta_{\max }$ of 5 (initial scheduling and up to 4 rescheduling cycles) and 1 (only initial scheduling - equivalent to RI).

To summarise, we run the execution batch of 150 workflows utilising two different scheduling schemes (new rescheduling technique and the previous RI technique), three different resource failure characteristics (shaky, normal, stable) and two different maximum replication sizes (two and four), summing up to over 1800 workflow runs, accumulating a total of tens of millions of CPU hours.

\subsection{Experimental Results}

While the pure full-ahead scheduling approach in our previous work on RI achieved a high level of fault tolerance for workflow executions, it proved to also generate large spikes in the execution time behavior of workflows in environments with a high fault rate. This is explained by the fact that the method is unable to readjust its fault tolerance strategy at runtime if the high fault rate 
made the chosen replication amount ineffective. Once this happens, the method has to fall back to resubmission, which leads to spikes in the workflow makespans that exceed the expected execution times by large amounts, deteriorating the QoS for the end-user. Our new method foresees potential situations leading to longer than expected workflow makespans and actively steers against this by adjusting the replication size at runtime. The ultimate goals of our approach are twofold. Firstly, we want to mitigate spikes in the makespan of workflows, thereby increasing the QoS delivered to the end user. Secondly, we want to increase the percentage of workflows that finish within the given soft-deadline.

Figure 2 and 3 show the cumulative distribution functions (CDF) of the successfully finished workflow executions with a maximum replication count $r e p_{\max }$ of 4 and 2, respectively, for all three fault characteristics: stable (a), normal (b) and shaky (c) and for both the new rescheduling technique and the previous RI approach. We define RES (shown on the horizontal axis) to be the ratio of the workflow makespan to the workflow soft-deadline $t_{d}$. RES values below 1 denote that a workflow has successfully completed before the given soft-deadline, while RES values larger than 1 denote that the given soft-deadline was overshot. A workflow with an RES of 1.0 has finished exactly at the given soft-deadline. The vertical axis shows the percentage of workflows that finished within a given RES. More concretely, assuming that the point $[0.8,60.0]$ belongs to the drawn function, then $60 \%$ of the workflows finished within an RES of 0.8 , or in other words, $60 \%$ of the workflows finished at least $20 \%$ faster than planned by the soft-deadline.

All the functions in Figures 2 and 3 exhibit a steep slope at an RES value of around 0.65. This is a result of workflows being executed entirely according to the initial full-ahead schedule, without experiencing faults that the initial replication setting could not compensate for. These workflows therefore did not have to resort to resubmission for their tasks, hence they were able to finish within the timespan initially expected by the first schedule.

To evaluate the improvements towards our first goal, the mitigation of large spikes in the workflow makespans, we compare the maximum RES value of both approaches. A lower maximum RES value represents a better mitigation of the makespan spikes. In the case of shaky resources and $r e p_{\max }=4$ (Figure 2(c)), the new rescheduling approach can finish all of the successfully executed workflows within an RES of 1.06. In contrast, the previous approach, RI with full-ahead scheduling, only leads to an RES value of 1.81 , which means that the workflow makespans overshot the soft-deadline by up to $81 \%$. For normal resources and $r e p_{\max }=4$ (Figure 2(b) , our new technique shows a $12 \%$ improvement in the maximum RES value from 1.17 to 1.05 , while in the stable resource case (Figure 2(a) it exhibits a slightly higher improvement, from 1.17 to 1.04 .

Figure [3] shows that our technique also manages to maintain an advantage when using a maximum replication count of 2 , although it is not able to mitigate the makespan spikes as well as in the case of $r e p_{\max }=4$. This is due to the limited fault tolerance potential when constrained to a maximum of two replicas for each task. The fact that the maximum RES value of our approach in the case 


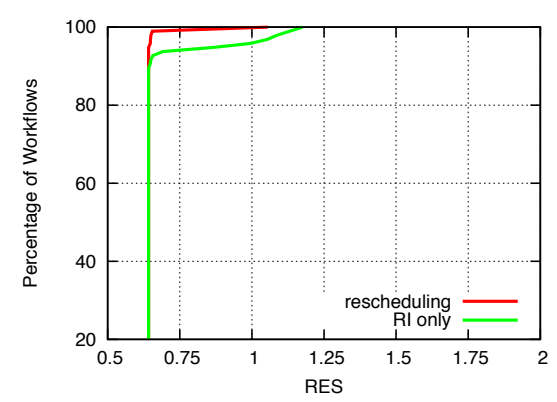

(a) Stable environment.

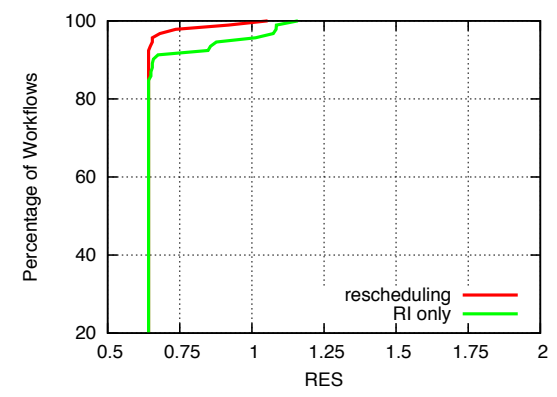

(b) Normal environment.

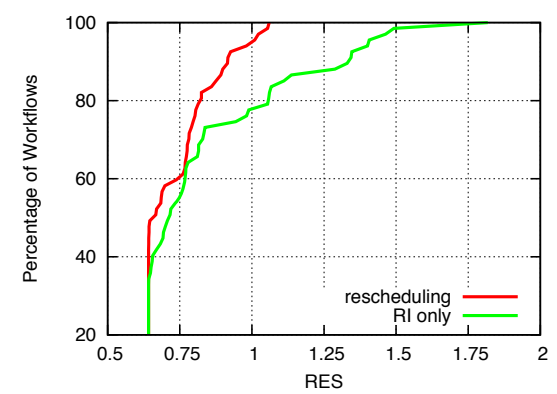

(c) Shaky environment.

Fig. 2. CDF of workflow executions with $r e p_{\max }=4$ and different fault properties

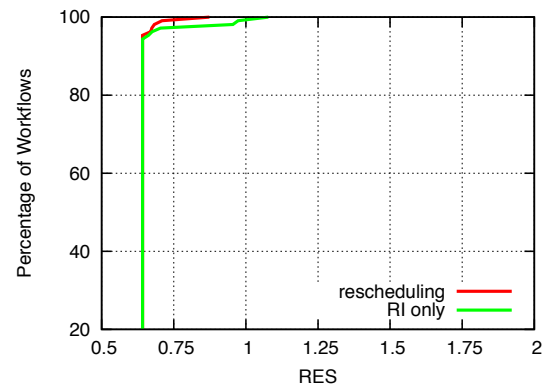

(a) Stable environment.

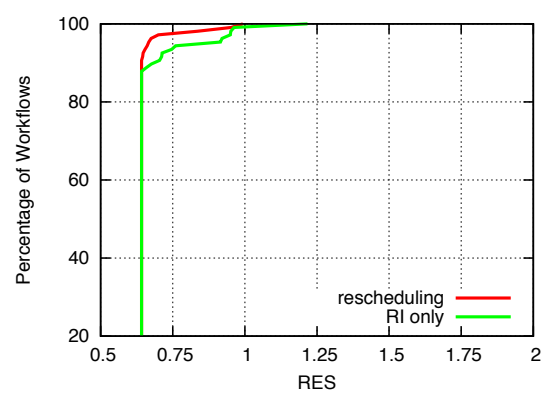

(b) Normal environment.

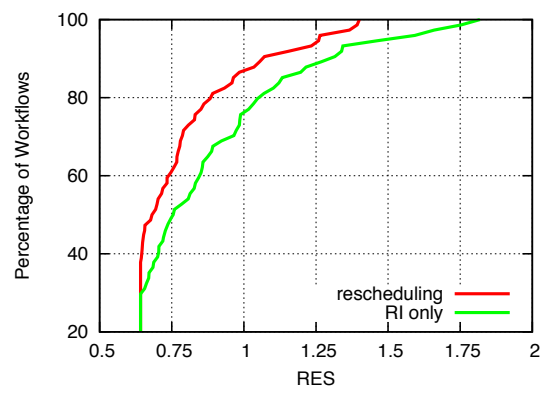

(c) Shaky environment.

Fig. 3. CDF of workflow executions with $r e p_{\max }=2$ and different fault properties

of stable and normal environments is smaller at $r e p_{\max }=2$ than at $r e p_{\max }=4$ is due to the lower execution success rate when using less replication.

To quantify the improvements towards the second goal, a higher percentage of workflows finishing within the given soft-deadline, we compare the vertical distance between the function plots of the two methods at an RES of 1.0. A higher distance represents a larger improvement towards our goal. Figure 2(c) shows that out of all the successfully executed workflows on shaky resources and a maximum replication count of 4 , our new technique finishes $95 \%$ within 
the soft-deadline, while the RI technique only manages to finish $78 \%$ within the deadline. For the normal resources (Figure 2(b)), the new approach finishes $99 \%$ of the workflows within the deadline, while RI achieves $95.5 \%$. On stable resources (Figure 2(a) , our proposed method still exhibits a slight improvement: $99.7 \%$, as opposed to $96 \%$ for the RI approach.

Similarly, in the case of $r e p_{\max }=2$ (Figure 3), our new technique consistently manages to finish a higher percentage of the workflows within the given deadline compared to the RI method. Despite the lower potential for dynamic adaptation with a maximum replication size of 2 as opposed to 4 in the previous cases, the new approach still manages to increase the percentage of workflows that meet the deadline from $76 \%$ to $87 \%$ on the shaky resources and from $99.3 \%$ to $100 \%$ on the normal and stable resources.

In conclusion, our experiments show that our newly proposed technique drastically reduces spikes in the makespans of workflow executions in faulty environments. Additionally, the remaining makespan spikes are of considerably shorter durations, up to $75 \%$, than the ones produced by the full-ahead scheduling RI approach. Finally, we demonstrated that our method is able to consistently meet soft-deadlines for a large percentage of the successfully executed workflows, even in unreliable environments.

\section{Conclusions}

We proposed in this paper a new method and algorithm to meet soft deadlines for workflow applications on highly distributed systems in the absence of failure models. To improve upon the problem of large makespan spikes and low Quality of Service in other approaches, our new method foresees possible deadline violations at runtime and takes appropriate measures to positively influence the execution of the remainder of the workflow. We achieve this by improving on our previous work, the RI heuristic, by introducing a new method for execution control as well as a rescheduling algorithm that is able to dynamically readjust the fault tolerance strategy at runtime.

We evaluated our method through comprehensive simulation using traces of three real applications executed in the Austrian Grid environment. Our results show that our approach meets all of the key goals: it strongly reduces spikes in the workflow makespans (by up to $75 \%$ ) and increases the percentage of workflows that finish within the soft-deadline (by up to $17 \%$ ).

Compared to related work, our approach does not rely on resource failure prediction which is difficult to achieve even with years of historic failure trace data of the target environment. Therefore, our approach has the advantage of immediate applicability in new, unknown, or even proprietary systems (i.e. commercial Cloud systems) where historical trace data is unavailable.

\section{References}

1. Blaha, P., Schwarz, K., Madsen, G., Kvasnicka, D., Luitz, J.: WIEN2k: An Augmented Plane Wave plus Local Orbitals Program for Calculating Crystal Properties, Institute of Physical and Theoretical Chemistry, TU Vienna (2001) 
2. Brandic, I., Pllana, S., Benkner, S.: Specification, planning, and execution of qosaware grid workflows within the amadeus environment. Concurrency Computat.: Pract. Exper. (January 2008)

3. Buyya, R., Murshed, M.: GridSim: a toolkit for the modeling and simulation of distributed resource management and scheduling for Grid computing. Concurrency and Computation-Practice \& Experience 14(13-15), 1175-1220 (2002)

4. Cotton, W.R., Pielke Sr., R.A., Walko, R.L., Liston, G.E., Tremback, C.J., Jiang, H., McAnelly, R.L., Harrington, J.Y., Nicholls, M.E., Carrio, G.G., McFadden, J.P.: RAMS 2001: Current status and future directions. Meteorology and Atmospheric Physics 82(1), 5-29 (2003)

5. Guo, L., McGough, A., Akram, A., Colling, D., Martyniak, J.: Qos for service based workflow on grid. In: Proceedings of UK e-Science 2007 All Hands Meeting (January 2007)

6. Iosup, A., Jan, M., Sonmez, O., Epema, D.: On the dynamic resource availability in grids. In: 2007 8th IEEE/ACM International Conference on Grid Computing, pp. 26-33 (2007)

7. Kandaswamy, G., Mandal, A., Reed, D.: Fault tolerance and recovery of scientific workflows on computational grids. In: 8th IEEE International Symposium on Cluster Computing and the Grid, CCGRID 2008, pp. 777-782 (2008)

8. Plachetka, T.: POVRAY - persistence of vision parallel raytracer. In: Computer Graphics International, pp. 123-129 (1998)

9. Plankensteiner, K., Prodan, R., Fahringer, T.: A new fault tolerance heuristic for scientific workflows in highly distributed environments based on resubmission impact. In: Fifth IEEE International Conference on e-Science, e-Science 2009, pp. 313-320 (December 2009)

10. Plankensteiner, K., Prodan, R., Fahringer, T., Kertesz, A., Kacsuk, P.: Faulttolerant behavior in state-of-the-art grid worklow management systems. Tech. Rep. TR-0091, Institute on Grid Information, Resource and Worklow Monitoring Services, CoreGRID - Network of Excellence (October 2007)

11. Topcuoglu, H., Hariri, S., Wu, M.: Performance-effective and low-complexity task scheduling for heterogeneous computing. IEEE Transactions on Parallel and Distributed Systems 13(3), 260-274 (2002)

12. Volkert, J.: Austrian grid: Overview on the project with focus on parallel applications. In: International Symposium on Parallel and Distributed Computing (2006)

13. Wieczorek, M., Siddiqui, M., Villazon, A., Prodan, R., Fahringer, T.: Applying advance reservation to increase predictability of workflow execution on the grid. In: Second IEEE International Conference on e-Science and Grid Computing (2006)

14. Yu, J., Buyya, R., Tham, C.: Qos-based scheduling of workflow applications on service grids. In: Proceedings of the 1st IEEE International ... (January 2005)

15. Zhang, Y., Mandal, A., Koelbel, C., Cooper, K.: Combined fault tolerance and scheduling techniques for workflow applications on computational grids. In: 9th IEEE/ACM International Symposium on Cluster Computing and the Grid, CCGRID 2009, pp. 244-251 (2009)

16. Zheng, T., Woodside, M.: Heuristic optimization of scheduling and allocation for distributed systems with soft deadlines. In: Computer Performance, pp. 169-181 (2003) 